\title{
SUMMARY FUNCTION ELASTICITY ANALYSIS FOR AN INDIVIDUAL-BASED SYSTEM DYNAMICS MODEL
}

\author{
Qian Zhang \\ School of Informatics and Computing \\ Indiana University \\ Bloomington, IN, 47408, USA
}

\author{
Nathaniel Osgood \\ Department of Computer Science \\ University of Saskatchewan \\ Saskatoon, SK, S7N5C9, Canada
}

\begin{abstract}
While eigenvalue elasticity analysis can offer insights into System Dynamics model behavior, such analysis is complicated, unwieldy and infeasible for larger models due to superlinear growth of the number of eigenvalueparameter as the number of stocks rises. To overcome these difficulties, we develop a summary function elasticity analysis method, which aids in analyzing the impact of a parameter on some global summary of the system state. A summary function defines a scalar field over state space summarizing the global state of a system. Summary function elasticity with respect to a parameter measures the ratio of the proportional change in the function to the proportional change in a parameter. We use an individual-based viral spread model to demonstrate that this new method offers greater simplicity than eigenvalue elasticity analysis while retaining most of its advantages. This method can be readily scaled to analyze impacts of parameters on larger-scale System Dynamics models.
\end{abstract}

\section{INTRODUCTION}

Eigenvalue elasticity analysis was first introduced by N. Forrester in 1982 (Forrester 1982) in the field of system dynamics. Past system dynamics research has applied this method to quantitatively analyze and identify significant parameters and loop structures in linear and simple nonlinear dynamical models with a small number of state variables (Saleh 2002, Saleh, Davidsen, and Bayoumi 2005, Goncalves 2006, Guneralp 2005, Guneralp 2006).

However, our early work found some important flaws of this method (Zhang and Osgood 2009). First, although eigenvalue elasticity analysis can be helpful for understanding the impact of parameter changes for simple nonlinear models with a small number of state variables, the method proves complicated, unwieldy and even infeasible when the number of state variables increases. For example, earlier work (Zhang and Osgood 2009) demonstrated such difficulties of analyzing an individual-based viral dynamic model with 3-person composing of 12 state variables. These difficulties are primarily due to increasing number of combinations of eigenvalues and parameters. This work also found that due to the variation of coefficients in the eigenvector solutions of a nonlinear dynamical model, the eigenvalue with the largest real part alone may not describe the dominant behavior of the system over a short period of time, and there might be several eigenvalues of the system Jacobian matrix that jointly determine the behavior pattern. In such cases, it is difficult to analyze eigenvalue elasticities to find significant parameters of the system. Finally, for an individual-based system dynamics model incorporating duplicated substructure (for example, several identical individuals represented with common substructure and sharing some parameter values), the eigenvalue elasticity method can easily fall prey to eigenvalue multiplicity problems.

To overcome the above difficulties of traditional eigenvalue elasticity analysis, we shift our attention from the eigenspace to the state space of a dynamical system and develop a summary function elasticity (sensitivity) analysis method with inspirations from the eigenvalue elasticity method. A summary function defines a scalar field over the state space which summarizes the overall states of a system at a particular time point. Eigenvalue sensitivity 


\section{Zhang and Osgood}

measures the ratio of the absolute change in an eigenvalue to the absolute change in a parameter; meanwhile eigenvalue elasticity is a measurement of the ratio of the proportional (e.g. percentage) change in the eigenvalue to the proportional (e.g. percentage) change in a parameter (Zhang 2008). Similar to the above definition of eigenvalue elasticity and sensitivity, the summary function elasticity (sensitivity) with respect to a parameter is defined as a measurement of the ratio of the proportional (absolute) change in the summary function to the proportional (absolute) change in a parameter. With these definitions, we can analyze the impact of a parameter on a system on some overall summary of the system states. In the remaining sections of this paper, we first give explicit mathematical definitions of a summary function and its elasticity and sensitivity. After that we apply these two new methods on analyzing an individual-based viral dynamics model with 30 persons to illustrate advantages of the method; and discuss each of these two methods respectively. Finally we draw conclusions.

\section{SUMMARY FUNCTION AND ITS ELASTICITY AND SENSITIVITY}

A System Dynamics model can be considered as a dynamical system in which the system state can be represented as a point in state space, each of whose axes corresponds to a particular state variable. Mathematically, the evolution of such a system can be studied with differential equations methods. To be specific, a System Dynamics model can be expressed with first order ordinary differential equations, in the form

$$
\dot{\mathbf{x}}(t)=\mathbf{f}\left(x_{1}(t), x_{2}(t), \cdots, x_{N}(t)\right) \mathbf{x}(t)+\mathbf{c}
$$

where $N$ is the number of state variables (stocks in System Dynamics) in the system and $\mathbf{f}$ is nonlinear functions of the state variables and $\mathbf{c}$ is a constant vector. The system begins to evolve with a set of initial conditions for each state variable at time $t_{0}$

As it is mentioned above, a summary function in the state space is defined as a scalar function summarizing the overall state of a system $\left(G\left(x_{1}, x_{2}, \cdots, x_{N}\right): \mathfrak{R}^{N} \mapsto \mathfrak{R}\right)$ at particular time points, e.g. in an infectious disease spread model, it can be the average viral load in population members, the total number of infected or infectious people, or cumulative mortality in the application field of epidemiology. In a fashion similar to the definition of eigenvalue sensitivity with respect to parameters, we define summary function sensitivity as

$$
g_{s}=\lim _{\Delta p \rightarrow 0} \frac{\Delta G}{\Delta p}=\frac{\partial G}{\partial p}
$$

And the summary function elasticity is defined as

$$
g_{e}=\lim _{\Delta p \rightarrow 0} \frac{\frac{\Delta G}{G}}{\frac{\Delta p}{p}}=\frac{\frac{\partial G}{G}}{\frac{\partial p}{p}}=\frac{\partial G}{\partial p} \frac{p}{G}=g_{s} \frac{p}{G}
$$

The derivative of a summary function over time can also describe the overall behavior of the system by indicating the rate of change of the summary function. Explicating this, we define the elasticity of the summary function's rate of change with respect to a parameter to show how much the rate of growth or decrease of a summary function could be affected by a small change in a parameter. In this study, this elasticity or sensitivity is called $\dot{G}$ elasticity or $\dot{G}$ sensitivity.

$$
\begin{gathered}
\dot{g}_{s}(p)=\lim _{\Delta p \rightarrow 0} \frac{\Delta \dot{G}}{\Delta p}=\frac{\partial \dot{G}}{\partial p} \\
\dot{g}_{e}(p)=\lim _{\Delta p \rightarrow 0} \frac{\frac{\Delta \dot{G}}{\dot{G}}}{\frac{\Delta p}{p}}=\frac{\frac{\partial \dot{G}}{\dot{G}}}{\frac{\partial p}{p}}=\frac{\partial \dot{G}}{\partial p} \frac{p}{\dot{G}}=\dot{g}_{s} \frac{p}{\dot{G}}
\end{gathered}
$$

Using the total differential, $\dot{G}$ could be written as

$$
\dot{G}=\frac{d G}{d t}=\frac{\partial G}{\partial t}+\sum_{i=1}^{N} \frac{\partial G}{\partial x_{i}} \dot{x}_{i}
$$


We note that each of the $\dot{x}_{i}$ is specified by the series of differential equations. With proper choice or construction of a summary function, the term $\frac{\partial G}{\partial t}$ and $\frac{\partial G}{\partial x_{i}}$ could be computed symbolically prior to the start of simulation. Based on these definitions, we can analyze the impact of a parameter on the system on some overall summaries of the system states. When there are high-level summary functions of clear interest, this method provides a more direct view than eigenvalue elasticity analysis for informing our understanding of the significance of parameters.

\section{APPLICATIONS ON AN INDIVIDUAL-BASED VIRAL DYNAMICS MODEL}

In this section, we will apply summary function elasticity and sensitivity to analyze an individual-based system dynamics model. To show the efficiency and convenience of this new methodology, we use the same framework of the individual-based model analyzed in our early work (Zhang and Osgood 2009), but change the population size of the model from 3 to 30 .

\subsection{Model Descriptions}

The individual-based immuno-epidemiological model we employ here describes the dynamics of viruses spreading within and between individuals at the level of cells. This micro-level epidemiological model provides a novel perspective from which to understand infectious diseases in terms of dynamical systems using characterizing mathematical tools such as differential equations. The purpose of research in this field is to reveal the basic laws that control the spread of infectious agents within an individual, their interactions with the immune system, and their responses to treatment (Nowak and May 2000). In this paper, this simple model of virus dynamics considers the populations of uninfected cells, infected cells, free virus particles, and the effect of CTL responses that can eliminate infected cells.

Based on the basic model for an individual, we follow an individual-based network model developed in (Vickers and Osgood 2007) with the assumption that interactions between individuals result in flow of free virus particles among those people. We use the same model of the individual-based viral dynamics model in (Zhang and Osgood 2009), which is followed the multi-individual model depicted in (Vickers and Osgood 2007). In this model, for a single individual there are four state variables: the population size of uninfected cells $x$, the population size of infected cells $y$, the number of free virus particles $v$, and the number of CTL $z$. For the number of free virus particles, each individual interacts with others in form of exchanging free virus $v$. The state equations of this model are shown as Equation 7, and its stock and flow structure is shown in Figure 1.

$$
\begin{aligned}
\dot{x_{i}} & =\lambda-d x_{i}-\beta x_{i} v_{i} \\
\dot{y_{i}} & =\beta x_{i} v_{i}-a y_{i}-p y_{i} z_{i} \\
\dot{v_{i}} & =k y_{i}-u v_{i}+\omega \sum_{i \neq j} \sigma_{i j} v_{j} \\
\dot{z_{i}} & =c y_{i} z_{i}-b z_{i}
\end{aligned}
$$

Where, $i, j=1, \cdots, P$ and $P$ is the size of population. The unit for $x, y, v$, and $z$ is cells, cells, virions, and CTL respectively. Explanations and initial settings of parameters are listed in Table $1 . \sigma_{i j}$ indicates whether the $i^{\text {th }}$ individual and the $j^{\text {th }}$ individual are connected. The values of this parameter in the model compose a connection matrix $\Sigma=\left(\sigma_{i j}\right)$ in the network, and we assume that this connection matrix is symmetric and $\sigma_{i i}=0$.

In this paper, we concentrate on an individual-based viral dynamics model with a relatively larger population size (30) than the model in our early work (population size 3) by summary function elasticity and sensitivity. The model with the 30 persons is similar to that with 3 persons except for presence of a larger population size and the presence of a different connection matrix $\left(\sigma_{i j}\right)(i, j=1, \cdots, 30)$. Figure 1 shows the network of connection among 30 persons in this model.

For such an immuno-epidemiological model, the motivations for the analysis of parameter impact on summary functions is several-fold. For an individual-based model with a large population, we believe that policy makers cannot limit their concerns to one or two individuals in the system, and their aim is control the disease on a macro-level. Another direct inspiration for our introduction of summary function elasticity and sensitivity, as we stated above, is the difficulty of applying eigenvalue elasticity methods to an individual-based model with a large population size. In contrast to our previous work (Zhang and Osgood 2009) which synthesized and recast findings from other contributions, the work presented in this section is novel to this research area. 


\section{Zhang and Osgood}

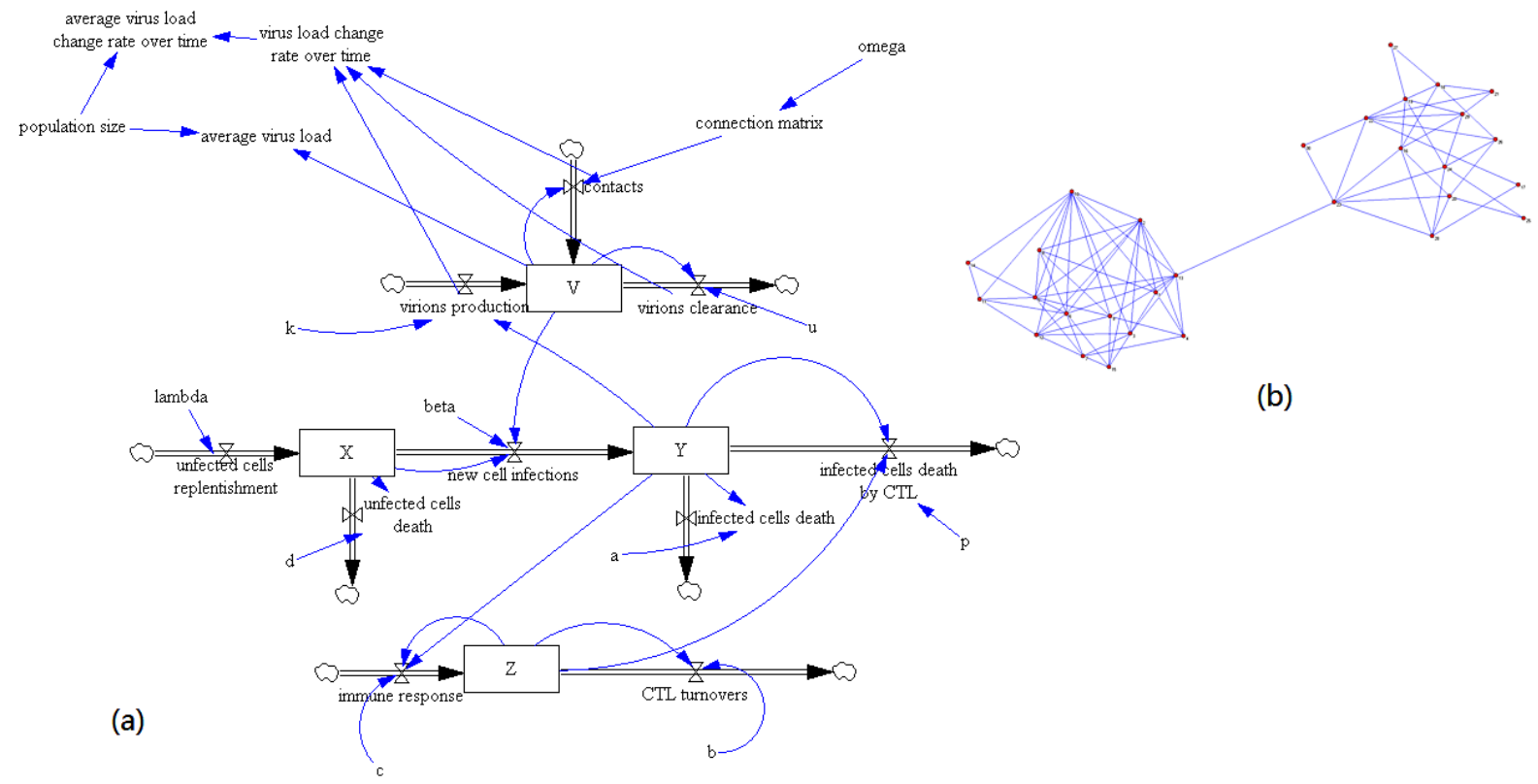

Figure 1: An individual-based viral dynamics model. (a): the stock-flow diagram of an individual-based immunoepidemiological dynamics model of viral spread. (b): the connection network

For the purpose of disease control, one possible target could be decreasing the amount of virus or the rate at which the quantity of viral particles grows on a level of the whole population. Thus we define a summary function $G$ to be the average viral load in the population. Because any change of a parameter cannot instantly influence the state variables, but the changing rate of state variables and of functions of state variables can be changed immediately, in following sections, we apply our analysis of the impact of parameter changes on the rate of change of summary functions. The summary function we study here is the rate of changing of the mean viral load: $\dot{G}$. If $P$ is the population size of the model, $G$ is defined as:

$$
G=\frac{1}{P} \sum_{i=1}^{P} v_{i}
$$

Table 1: Parameter settings of an individual-based viral dynamics model.

\begin{tabular}{|c|c|c|c|}
\hline Parameter & Full Name & Value & Units \\
\hline$\beta$ & the rate of uninfected cells to be infected & $10^{-5}$ & $1 /$ day $\cdot$ virions \\
\hline$k$ & the rate of infected cells to produce free virus & 3 & virions/day·cells \\
\hline$d$ & the death rate of uninfected cells & 0.1 & day $^{-1}$ \\
\hline$u$ & the death rate of free virus & 3 & day $^{-1}$ \\
\hline$a$ & the death rate of infected cells & 0.5 & $\mathrm{day}^{-1}$ \\
\hline$\lambda$ & the replenishing uninfected cell rate & $10^{5}$ & cells/day \\
\hline$p$ & the rate of infected cells to be eliminated by the CTL response & 1 & 1/day.CTLs \\
\hline$c$ & the production rate of $\mathrm{CTL}$ & 0.7 & 1/day.CTLs \\
\hline$b$ & the death rate of $\mathrm{CTL}$ & 0.05 & $\mathrm{day}^{-1}$ \\
\hline$\omega$ & the connection weight & $10^{-6}$ & 1/day \\
\hline$\sigma_{i, j}$ & the entry of the connection matrix & 1 or 0 & - \\
\hline
\end{tabular}


According to Equation 6, we have

$$
\dot{G}=\frac{\partial G}{\partial t}+\sum_{i=1}^{P} \frac{\partial G}{\partial x_{i}} \dot{x}_{i}=0+\sum_{i=1}^{P} \frac{\partial G}{\partial x_{i}} \dot{x}_{i}
$$

From the definition of $G$ in Equation 8, we have

$$
\begin{gathered}
\frac{\partial G}{\partial x_{i}}=\frac{\partial G}{\partial y_{i}}=\frac{\partial G}{\partial z_{i}}=0 \\
\frac{\partial G}{\partial v_{i}}=\frac{1}{P}
\end{gathered}
$$

With Equation 7, we have

$$
\dot{G}=\frac{1}{P} \sum_{i=1}^{P} \dot{v}_{i}=\frac{1}{P} \sum_{i=1}^{P}\left(k y_{i}-u v_{i}+\omega \sum_{i \neq j} \sigma_{i j} v_{j}\right)
$$

From Equation 10, it can be observed that the summary function $\dot{G}$ depends on three parameters: $k$ (the rate at which infected cells produce free virus), $u$ (the death rate of the free virus), and $\omega$ (the connection weight, dictating the rate of viral transmission between neighbors). In this section, we analyze the summary function elasticity with respect to $u$ and $\omega$. We do so because in practice it may be possible to perturb the parameter $u$ by biological or medical treatment, and the parameter $\omega$ by interventions focused on risk behavior modification, hygiene, etc.

With Equation 4 and Equation 10, the sensitivity of $\dot{G}$ with respect to parameters $u$ and $\omega$ is as follows:

$$
\begin{gathered}
\dot{g}_{s}(u)=-\frac{1}{P} \sum_{i=1}^{P} v_{i} \\
\dot{g}_{s}(\omega)=\frac{1}{P} \sum_{i=1}^{P} \sum_{i \neq j} \sigma_{i j} v_{j}
\end{gathered}
$$

We are now able to analyze the elasticity of the summary function $\dot{G}$ with Equation 11, Equation 12 and Equation 5 . From Equation 5, we have

$$
\dot{g}_{e}(p)=\lim _{\Delta p \rightarrow 0} \frac{\frac{\Delta \dot{G}}{\dot{G}}}{\frac{\Delta p}{p}}
$$

In this section, we also calculate anticipated (theoretic) changes in $\dot{G}$ resulting from changing a parameter using the following approximations:

$$
\begin{gathered}
\frac{\Delta \dot{G}}{\dot{G}} \approx \dot{g}_{e}(p) \frac{\Delta p}{p} \\
\Delta \dot{G} \approx \dot{g}_{e}(p) \dot{G} \frac{\Delta p}{p}
\end{gathered}
$$

Equation 14 tells us that the proportional change of $\dot{G}$ approximately equals the multiplication of the elasticity of $\dot{G}$ with respect to a parameter $p$ and the proportional change of $p$. In this study, one purpose of parameter perturbations is to decrease $\dot{G}$ so that the average free viral particles $v$ could decrease faster or increase more slowly (i.e., $\Delta \dot{G}<0$ ). Whether we should increase or decrease the parameter $p$ to accomplish this depends on values of both the elasticity and $\dot{G}$. 


\subsection{Summary Function Elasticity Analysis of an Individual-based Viral Dynamics Model with 30 Persons}

\subsubsection{Summary Function Elasticity with Respect to the connection weight $\omega$}

The $\dot{G}$ elasticity with respect to $\omega$ over time for the 30-person model is presented in Figure 2 . The elasticity has large values after time $=300$, especially after time $=500$. Policy makers will prefer to intervene when the disease first breaks out in order to decrease the cost brought by disease spread. Although the summary function elasticity has large values in the late stage of the system evolution, it also attains relatively high absolute values in the early stage of the system evolution. From this figure we could know that there are peak values from time $=0$ to time $=100$ when the system first evolves, though these peaks are almost invisible compared with significant peaks in the later stages.
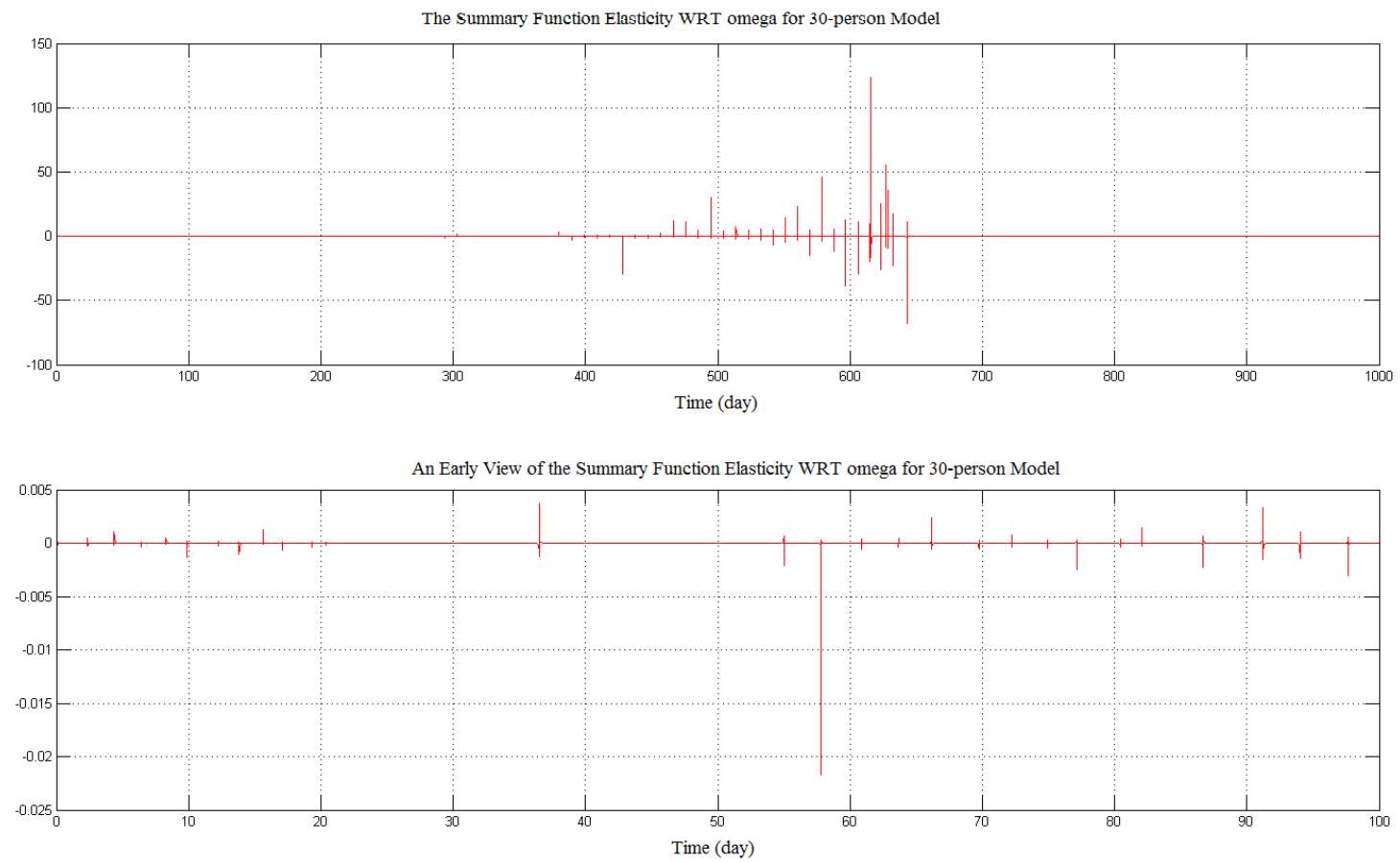

Figure 2: The summary function elasticity with respect to $\omega$ (the connection weight) for an individual-based viral dynamics model with 30 persons and its early view.

Three time points with apparent peak values in the time period from time $=0$ to time $=100$ are selected to perturb the parameter $\omega$ separately for the 30-person model. The summary function elasticity with respect to $\omega$ arrives at a peak value at time $=4.37$ with the value of $1.029 \times 10^{-3}$, and another peak value at time $=9.89$ with the value of $-1.374 \times 10^{-3}$ and the third peak value at time $=36.51$ with the value of $3.673 \times 10^{-3}$ when other values of the elasticity are on the order of magnitude of $10^{-5}$ or smaller. $\omega$ was decreased to $10 \%$ of the original value at these time points. With simulations, we found that initially such perturbations change the trajectory of $\dot{G}$, and the proportional change of $\dot{G}$ is not far from the prediction derived from Equation 15. However, in the long run, the perturbation at time $=9.89$ has similar influences on $\dot{G}$ to the perturbation at time $=36.51$. A control experiment is added to decrease $\omega$ by $90 \%$ at time $=5.43$ when the elasticity of the summary function with respect to $\omega$ is $1.864 \times 10^{-6}$. In a very short period of time after changing $\omega$, the proportional change of $\dot{G}$ at time $=5.43$ is indeed much smaller than that at time $=4.37$, but later on the differences of the proportional changes at two time points become less notable.

When approaching the endemic equilibrium, $\dot{G}$ approaches to zero no matter the parameters are perturbed or not. As it was noted in (Zhang 2008), for an individual-based model such as that here, the connection weight does not greatly influence the position of the endemic equilibrium because of its relatively low value. Here we also observe that the average virus loads near the endemic equilibrium vary little with perturbations of $\omega$. 


\subsubsection{Summary Function Elasticity with Respect to the death rate of the free virus $u$}

The impact of the $\dot{G}$ elasticity with respect to $u$ over time for the 30 people model is similar to that to $\omega$. Because the parameter $u$ can be increased by biological or medical treatment and because the purpose of perturbation is to decrease the summary function $\dot{G}$, we choose the time points when the elasticity is negative, and thus the large elasticity here means the negative values with large absolute values. Similar to the $\dot{G}$ elasticity with respect to $\omega$, the summary function elasticity with respect to $u$ attains relatively high absolute values in the early stage of the system evolution. There are peak values from time $=0$ to time $=100$ when the system first evolves and $\dot{G}$ has large values, though these peaks are almost invisible compared with significant peaks in the late stages, as described in Figure 3.
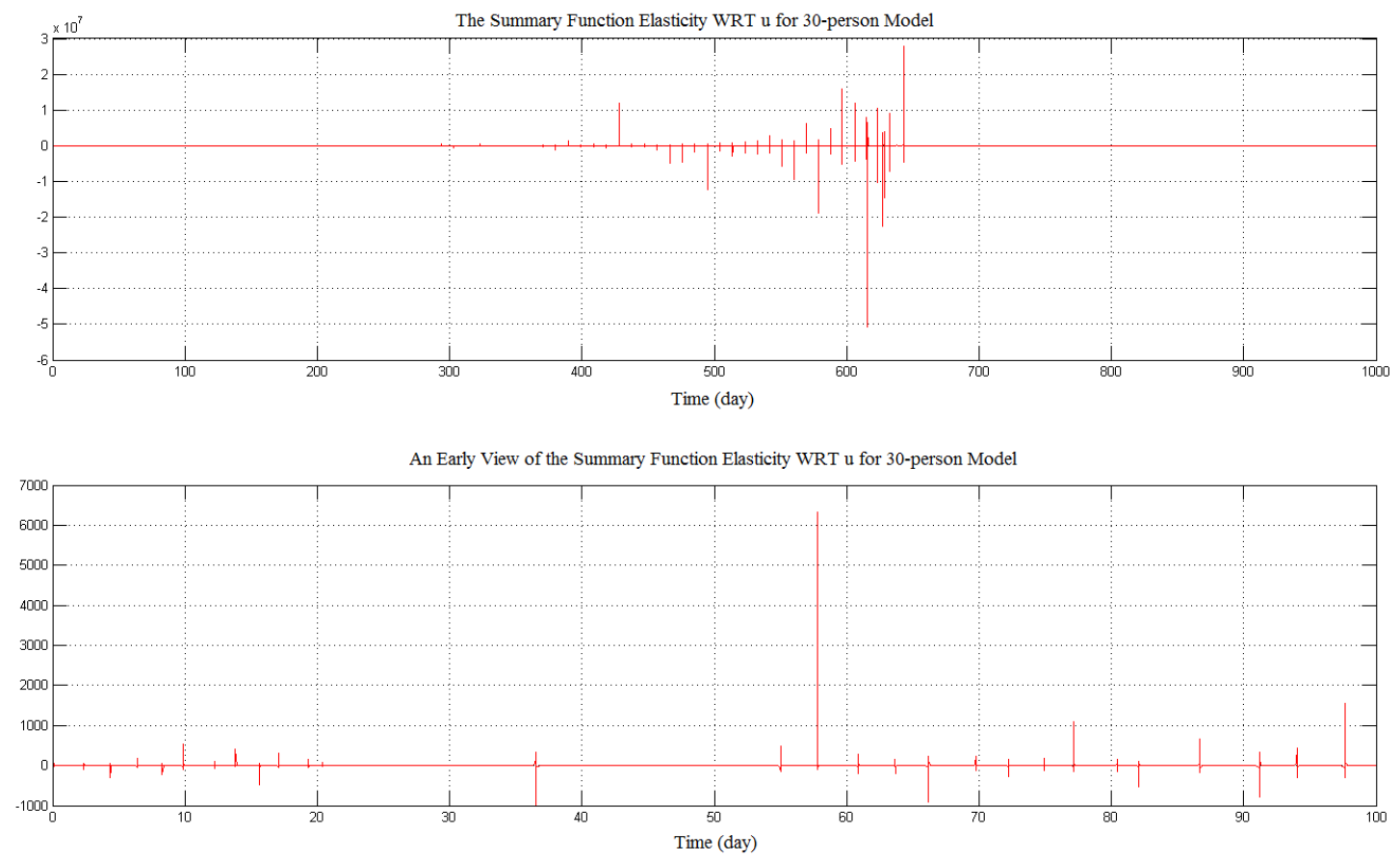

Figure 3: The summary function elasticity with respect to $u$ (the death rate of the free virus) for an individual-based viral dynamics model with 30 persons and its early view.

The summary function elasticity with respect to $u$ arrives at the peak value at the same time points as the elasticity of $\omega$ : time $=4.37$, time $=9.89$ and time $=36.51$, while the values of the elasticities are on the order of magnitude of $10^{1}$ or below at most of other time points during the early stage. We perturb $u$ to increase it by $10 \%$ at these time points so that $\Delta \dot{G}<0$. Shortly after the perturbations, the increment of $u$ at a time point of a high summary function elasticity could produce a significant alteration of $\dot{G}$ in a very short time period after the perturbation, i.e. the proportional change in a very short period after altering $u$ produced by the perturbation at time $=36.51$ is larger than those changes generated by the perturbation at time $=4.37$ and time $=9.89$.

A control experiment for the perturbation of $u$ is performed at time $=5.43$, when $\dot{G}=3.00334 \times 10^{-1}$, with the value of the summary function elasticity $-9.089 \times 10^{-1}$. As was predicted, immediately after the perturbation, $\frac{\Delta \dot{G}}{\dot{G}}$ is much smaller than that in the case of the perturbation at time $=4.37$ or at time $=9.89$. However, when the state variables approaches to equilibrium, because $G$ is associated with state variables, its changing rate $\dot{G}$ tends to be zero. In the long-term, trajectories converge and the perturbation of the parameter at any time point should result in similar behaviors of $\dot{G}$.

\subsubsection{Discussion}

In the above section, we can see that a perturbation at a time point when the summary function elasticity is high can result in a significant proportional change of $\dot{G}$ shortly after the perturbation. But over a long period, such perturbation at time points with high elasticity may not produce more notable proportional changes of $\dot{G}$. For the 
model at hand, the summary function elasticity usually exhibits larger deviations in the late stages of the system evolution. From Equation 5, we have $\dot{g}_{e}(p)=\frac{\partial \dot{G}}{\partial p} \frac{p}{G}$. With a given expression of $\frac{\partial \dot{G}}{\partial p}$ and the value of $p$, the elasticity is large when $\dot{G}$ is small. Thus for the current model (which approaches the endemic equilibrium) the elasticity has peak values in the late stages when the summary function has very small values. As it was noted in (Zhang 2008), changing parameters $\omega$ or $u$ by a small amount alters the position of the endemic equilibrium but does not alter the stability of the endemic equilibrium. Any perturbation of a parameter would produce similar trajectories of the summary function in the long-term when the system is near the endemic equilibrium. Therefore, the summary function elasticity analysis is only effective in a short period of time after the perturbation, during which the linear approximation used for the calculations remains valid.

In a short period after the perturbation, the proportional changes of $\dot{G}$ follow their theoretical values, i.e., a large $\dot{G}$ elasticity could produce a significant proportional change of $\dot{G}$. Based on Equation 15, we know that the absolute change of $\dot{G}$ for a given change of a parameter depends on both the elasticity and the value of $\dot{G}$, and thus it is possible that a perturbation of a parameter at a time point when the elasticity is low can result in an apparent absolute change of $\dot{G}$ if $\dot{G}$ has a high value at that time. Therefore, because of the large values of $\dot{G}$ in the early period of time, a small change of a parameter can generate a significant alteration of the behavior of $\dot{G}$, though the elasticities at the time points when the perturbation of the parameter is performed have small values.

A large value of the summary function elasticity cannot guarantee a desired result over a relatively long time. One possible reason is that for a nonlinear system its Jacobian matrix is changing over time. Great local proportional changes might become less significant because the structure of the system is changing. Another reason might be the accumulation or offset of the effects of changes of the parameter in later time points when the elasticity is high or low. Thirdly, because the trajectory is altered, the elasticity of the summary function with a parameter could be changed if the value of this parameter is altered, therefore our perturbation of a parameter in a long time may neglect such changes of the elasticity. At last, because trajectories of state variables converge towards endemic equilibrium, the change rate of the summary function approaches zero, the trajectory converges, and any perturbation of a parameter will not change $\dot{G}$ in a long-term.

It is worth emphasizing that because the summary function elasticity of a parameter is dimensionless, it is helpful for indicating the significance of a parameter on the system. The $\dot{G}$ elasticity with respect to $u$ is much larger than that to $\omega$, and thus the small perturbations of $u$ (10\% increased) produce more significant changes of the summary function that the large perturbations of $\omega$ (90\% decreased).

Because the summary function elasticity is defined as the ratio of proportional changes of the summary function to proportional changes of parameters, as Equation 5 shows, when the value of the summary function is near zero, the value of the elasticity can be extremely high. We conjecture (but have not demonstrated) that this is the reason for the impulse-like values shown in Figure 2 and Figure 3. Although the proportional changes of the summary function with perturbations of parameters at time points with those impulse-like elasticity values could be large, the absolute changes of the summary function is usually very small because of its near-zero values. Practically, it is less meaningful to change the summary function with tiny absolute changes at time points when its values are close to zero. There is a distinct risk of zero or near-zero values in the denominator of the eigenvalue elasticity can distract us from more practically important leverage points for changing system behavior.

\subsection{Summary Function Sensitivity Analysis of an Individual-based Viral Dynamics Model with 30 Persons}

In the last section, we saw that the rate of change in summary function, $\dot{G}$, has great influence on the value of the summary function elasticity. Motivated by that observation, in this section, we focus on the summary function sensitivity with respect to a parameter. Recall from Equation 4, we have

$$
\begin{gathered}
\dot{g}_{s}(p)=\lim _{\Delta p \rightarrow 0} \frac{\Delta \dot{G}}{\Delta p} \\
\Delta \dot{G} \approx \dot{g}_{s}(p) \Delta p
\end{gathered}
$$

From Equation 17 we know that the absolute change of $\dot{G}$ approximately equals the product of the sensitivity of $\dot{G}$ and the absolute change of the parameter $p .{ }^{1}$ Thus we know that the summary function sensitivity indicates

\footnotetext{
${ }^{1}$ An absolute change of $p$ here means $\Delta p=p_{\text {new }}-p$, and an absolute change of $\dot{G}$ is $\Delta \dot{G}=\left.\dot{G}\right|_{p_{\text {new }}}-\left.\dot{G}\right|_{p}$.
} 
how much the summary function changes in absolute terms when the parameter changes by a given absolute change amount. Because a high summary function sensitivity indicates a large change to $\dot{G}$ in response to a given change in a parameter regardless of the value of $\dot{G}$, we investigate here whether it might yield greater insight into leverage points than does the summary function elasticity.

\subsubsection{Summary Function Sensitivity with Respect to the connection weight $\omega$}

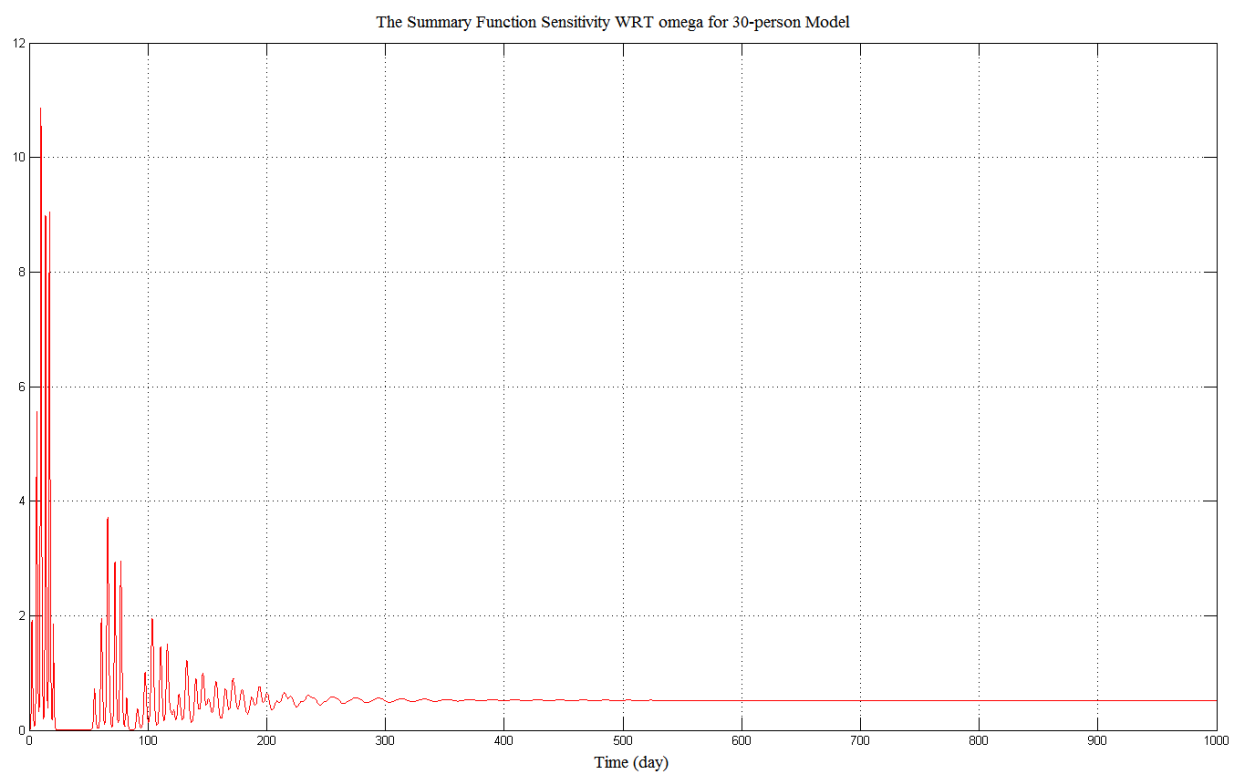

Figure 4: The summary function sensitivity with respect to $\omega$ (the connection weight) for an individual-based viral dynamics model with 30 persons.

$\dot{G}$ sensitivity with respect to $\omega$ (the connection weight) is shown in Figure 4. From this figure, we can learn that the sensitivity over time is positive and that before time $=100$, the $\dot{G}$ sensitivity attains apparent peaks with large values, especially between time $=0$ and time $=50$. The first peak of the value of the elasticity appears at time $=2.37$ with the value of 1.9239 , the second peak appears around time $=6.39$ when the value is 5.569 , and the third peak is at time $=9.87$ with the value of 10.8567 .

We now test new perturbations of parameters affecting $\dot{G}$. We decrease $\omega$ by $90 \%$ as we did in the summary function elasticity analysis. In an immediate period of time after perturbing the parameter, the absolute change of $\dot{G}$ approximates its theoretical value, which can be predicted by Equation 17. The short-term absolute change of the function is proportional to the summary function sensitivity at the time point when the parameter is decreased. But the long-term absolute changes produced by the perturbations of $\omega$ at time points with distinct differences of the values of sensitivities are approximately same because the system is approaching the endemic equilibrium, whose position does not depend on the parameter $\omega$.

\subsubsection{Summary Function Sensitivity with Respect to the death rate of the free virus $u$}

Figure 5 shows the $\dot{G}$ sensitivity with respect to $u$ (the death rate of the free virus) over time for a 30-person model. In this figure, the value of the sensitivity is negative over time, and it is quite large in the early stage of the system evolution. The first peak of the value of the sensitivity appears at time $=2.37$, with the value of -0.1489 , the second peak appears at time $=6.41$ which is -0.9593 , and the third peak is at time $=9.89$ with the value of -1.3925 . We change $u$ by $10 \%$ of the original value (increasing $u$ by 0.3 ), at these three time points to try to change the behavior of the summary function.

During a small window frame of time the values of the absolute changes of $\dot{G}(\Delta \dot{G})$ are close to the product of the summary function sensitivity and the absolute change of $u$. The absolute changes of $\dot{G}$ with a perturbation at time $=9.89$ (the time point with the highest absolute value of sensitivity in the experiments) are larger than the 


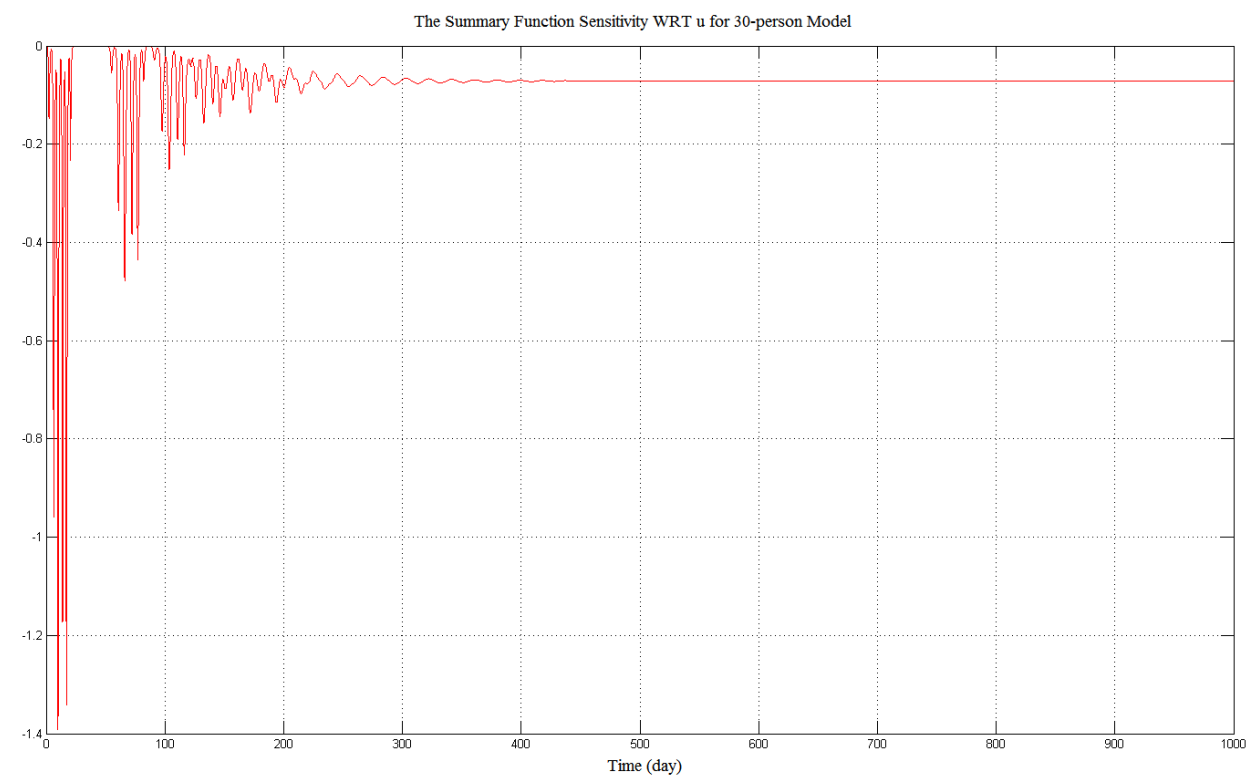

Figure 5: The summary function sensitivity with respect to $u$ (the death rate of the free virus) for an individual-based viral dynamics model with 30 persons.

changes with a perturbation at time $=6.41$ (the time with the second largest absolute value of sensitivity), which are greater than the absolute changes with a perturbation at time $=2.37$, because $\left.\dot{g}_{s}(u)\right|_{\text {time }=9.89}>\left.\dot{g}_{s}(u)\right|_{\text {time }=2.37}$. In a longer time, especially when the system approaches to the endemic equilibrium, the changes of the parameter $u$ could enlarge or shrink the magnitude of the oscillation of the values of $\dot{G}$; however, because the system asymptotically approaches an equilibrium, $\dot{G}$ asymptotically goes to zero, and cannot be altered by any change of the parameter.

\subsubsection{Discussion}

The above two sections suggest that summary function sensitivities are effective for indicating promising times for policy makers to perform control over disease spread in a short period of time. Because the summary function sensitivities are not affected by the influence of changes of the summary function values, this method could indicate the early time points when the $\dot{G}$ is sensitive to parameters. The perturbation of the parameter at time point when the sensitivity is large can locally change the trajectory of the summary function as the summary function sensitivity indicates, but the large sensitivity is neither necessary nor a sufficient condition of significant changes of the summary function in a long period of time. However, from the above sections, we can find that the early control of parameters at time points when the summary function sensitivities are large can alter the behavior of the summary function. Practically, it suggests that policies performed in the early stage when the system is far from equilibrium have great influences on the system behaviors. On the disadvantage side, because the summary function sensitivity is not dimensionless, we cannot use it to compare the relative importance of parameters for the system, if those parameters are associated with different dimensions.

\section{CONCLUSION}

Inspired by traditional eigenvalue elasticity and sensitivity analysis for nonlinear systems, we developed summary function based analysis to discover the influences of the parameters on the summary functions associated directly on state variables for infectious disease models. By applying this new method to a summary function over an individual-based viral Dynamics model with a relatively large population, we found that in a short period of time after the perturbation of a parameter at a time point when the elasticity or the sensitivity is high, the changes of the summary function are notable. Practically, this method is effective to inform the control of disease spread in a short time, such as in the period of the disease outbreak. In addition, similar to eigenvalue elasticity, summary function 
elasticity is also dimensionless, which means it is possible for us to apply such method to compare the importance of parameters for a model. In the background of public health modeling, such methods offer the potential for aiding policy makers in finding out crucial factors for disease control in a short period of time, especially during the outbreak of an infectious disease. Furthermore, different from the eigenspace-based analysis method, the number of state variables does not influence model analysis with the summary function elasticity or sensitivity; and this new method gets rid of eigenvalue multiplicity problems that can seriously complicate the analysis of models where each individual is identical.

However, globally the perturbations of a parameter cannot reliably determine how changing a parameter can affect $\dot{G}$ over a long period of time. This reflects the fact that for a nonlinear system, because local attributes, such as eigenvalues and eigenvectors of Jacobian matrix, vary over time, we are unable to determine global attributes of the system behaviors for long period of time. Around the endemic equilibrium, the behavior of the summary function is only strongly dependent on parameters related with the endemic equilibrium.

Therefore, for an individual-based viral dynamics model with a large population, if our attention focuses on immediate disease control during a short time period around a disease outbreak, the summary function elasticity and sensitivity analysis methods can be effective and efficient to predict the importance of parameters and the time points at which to change influential parameters. With an appropriate choice of a summary function, policy makers may be able to better control the severity of the infectious disease spread over the whole population. Similarly, this summary function based method can also be applied to short-term analysis of nonlinear individual-based System Dynamics models or models with a large number of state variables in other application backgrounds. But in the long run, such method is not able to effectively predict the behavior changes. For such cases, the numeric or symbolic analysis around endemic equilibria, as well as other methods could help us to better anticipate the long-term behavior patterns and parameter sensitivities, which is worth further research.

\section{REFERENCES}

Forrester, N. 1982. A dynamic synthesis of basic macroeconomic theory: Implacations for stabilization and policy analysis. Ph. D. thesis, MIT, Cambridge, MA.

Goncalves, P. 2006, July. Eigenvalue and Eigenvector Analysis of Dynamic Systems. In Proc. The 24th International Conference of the System Dynamics Society, ed. A. Größler, E. A. J. A. Rouwette, R. S. Langer, J. I. Rowe, and J. M. Yanni. Nijmegen, Netherland: The System Dynamics Society: The System Dynamics Society.

Guneralp, B. 2005, July. Progress in Eigenvalue Elasticity Analysis as a Coherent Loop Dominance Analysis Tool. In Proc. The 23rd International Conference of the System Dynamics Society, ed. J. D. Sterman, N. P. Repenning, R. S. Langer, J. I. Rowe, and J. M. Yanni, online. Boston, MA: The System Dynamics Society: The System Dynamics Society.

Guneralp, B. 2006. Exploring structure-behavior relations in nonlinear dynamic feedback models. Ph. D. thesis, University of Illinois at Urbana-Champaign.

Nowak, M. A., and R. M. May. 2000. Virus dynamics: Mathematical principles of immunology and virology. Oxford University Press.

Saleh, M. 2002. The Characterization of Model Behavior and its Causal Foundation. Ph. D. thesis, University of Bergen, Bergen, Norway.

Saleh, M., P. Davidsen, and K. Bayoumi. 2005, July. A Comprehensive Eigenvalue Analysis of System Dynamics Models. In Proc. The 23rd International Conference of the System Dynamics Society, ed. J. D. Sterman, N. P. Repenning, R. S. Langer, J. I. Rowe, and J. M. Yanni, online. Boston, MA: The System Dynamics Society: The System Dynamics Society.

Vickers, D., and N. Osgood. 2007. A Unified Framework of Immunological and Epidemiological Dynamics for the Spread of Viral Infections in a Simple Network-based Population. Theoretical Biology and Medical Modelling Volume 4 (49): doi:10.1186/1742-4682-4-49.

Zhang, Q. 2008. Application and Evaluation of Local and Global Analysis for Dynamic Models of Infectious Disease Spread. Master's thesis, Department of Computer Science, University of Saskatchewan, Canada.

Zhang, Q., and N. Osgood. 2009, July. Local Analysis of Individual-based Viral Dynamic Models with Eigenspace and Eigenvalue Elasticity Analysis. In Proc. The 27th International Conference of the System Dynamics Society, ed. A. Ford, D. N. Ford, and E. G. Anderson, online. Albuquerque, NM: The System Dynamics Society: The System Dynamics Society. 


\section{AUTHOR BIOGRAPHIES}

QIAN ZHANG is a PhD student in Center for Complex Networks and Systems Research at School of Informatics and Computing, Indiana University Bloomington. She received MSc. from Department of Computer Science in University of Saskatchewan in 2008. Her current research interest is in the area of epidemiological modeling including local-level modeling with System Dynamics and agent-based system and global-level modeling with stochastic process and complex networks. She also works on dynamics of complex networks. Her email address is $<$ zhang31@indiana.edu>.

NATHANIEL OSGOOD is an Assistant Professor in the Department of Computer Science and Associate Faculty in the Department of Community Health \& Epidemiology, School of Public Health and Division of Bioengineering at the University of Saskatchewan. Dr. Osgood's work focuses on providing tools to inform understanding of population health trends and health policy tradeoffs. This work includes both methodological and applications components. The methodological components seek to advance the art of model building through improved formalisms, algorithms, software tools, and design guidelines. Dr. Osgood is also involved in the design of wireless ambulatory sensing mechanisms to provide data for such models. On the application side, Dr. Osgood works closely with cross-disciplinary teams on several team-based projects applying mathematical \& computational modeling to address urgent public health challenges in both the chronic and infectious disease areas. He has a particular interest in projects related to the health of Aboriginal peoples. Dr. Osgood received his PhD in 1999 from MIT's Department of Electrical Engineering and Computer Science. Prior to joining the U of S faculty, he worked for many years in a number of academic and industry positions, including on industry \& academic projects applying modeling to tobacco and environmental epidemiology, health informatics, and multi-framework modeling for natural resource policy-making. His email address is <osgoodecs.usask.ca>. 